\title{
Study of Urinary Aminopeptidase
}

\author{
Takeshi ONo \\ Yawata Seitetsu Hospital Internal Medicine
}

In the measurement of LAP activity, this enzyme activity of kidney tissue is much higher than the one of any other organs.

LAP level of cortex is higher than LAP level of medulla, glomerular LAP level is low, and proximal tubular epithelial cells have markedly increased this enzyme activity.

Elevated level of urinary LAP was recognized in patients with malignant tumor, nephrotic syndrome and acute inflammation, and also some of the patients with diabetes mellitus had an elevated level of LAP activity.

In follow-up observation of the course of aminonucleoside nephrosis rats, urinary LAP activity began to increase at the 7 th day of the course.

In children with acute nephritis, there was not a greater increase in level of urinary LAP activity. But in some of adult patients with nephritis, urinary LAP showed a moderately elevated level.

As a result of the following investigations, it is suggested that the origin of urinary LAP is kidney tissue LAP.

1. In the electrophoretical findings of isozyme, kidney LAP identified with urinary LAP and serum LAP differed from urinary LAP.

2. In group of patients with miscellaneous diseases, the correlation between urinary LAP level and serum LAP level was not recognized.

3. From the histological findings of aminonucleoside nephrosis rats and from the LAP staining views of epithelial-cell casts in the urinary sediments of nephrotic patients, one might presume that urinary LAP increase is due to damage of kidney tubular cells.

As shown above, it is suggested that urinary activity of LAP is related primarily to renal-tubule cell damage and not to glomerular injury. 